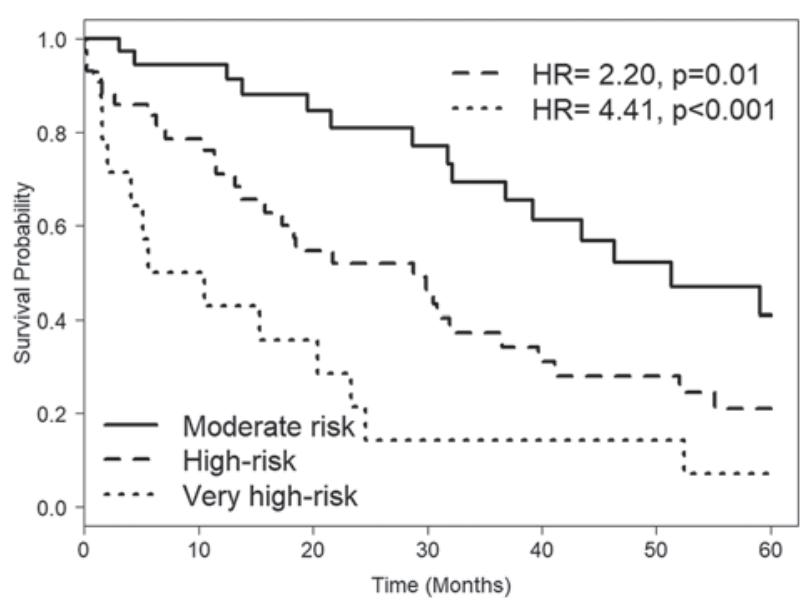

Abstact S55 Figure 1 Derivation cohort - ILD-PH prognostic score.

right heart catheterisation (RHC) for suspected ILD-PH. Predictors of prognosis were evaluated in their ability to predict mortality using Cox proportional hazard analysis. A prognostic model was developed and tested in a derivation cohort and tested in a separate validation cohort.

Results 180 patients with confirmed $\mathrm{PH}$ formed the derivation cohort (mean pulmonary arterial pressure (mPAP) at RHC 37 $\pm 9 \mathrm{mmHg} ; 50 \%$ male). At baseline, the strongest predictor of mortality was the underlying ILD diagnosis, with idiopathic pulmonary fibrosis or chronic hypersensitivity pneumonitis strongly associated with mortality (hazard ratio (HR):3.58, $\mathrm{p}<0.001)$. A relative decline in forced vital capacity (FVC) of $10 \%$ at $12-24$ months after RHC predicted mortality (HR:3.20, p=0.001), and an increase in BNP at 12-24 months was also associated with mortality (HR:2.27, $\mathrm{p}=0.005)$. A prognostic model combining baseline and longitudinal change risk stratified patients into very-high risk, highrisk and moderate risk groups. In the derivation cohort, the high-risk group had a HR of $2.20 \quad(p=0.01)$, and the very high-risk group a HR of $4.40(\mathrm{p}=0.001) .50$ patients with confirmed PH made up the validation cohort (mPAP 37 $\pm 9 \mathrm{mmHg} ; 46 \%$ male). The high-risk group had a HR of $3.60(\mathrm{p}=0.01)$ and the very high-risk group a HR of 8.17 $(\mathrm{p}<0.001)$.

Conclusion A simple prognostic score using longitudinal change in FVC and BNP powerfully predicts mortality in ILD-PH, and could be used to prognosticate and help prioritise precious organ allocation in this challenging population.

\section{S56 THE IMPACT OF AZITHROMYCIN IN IDIOPATHIC PULMONARY FIBROSIS}

O Alzaher, C Macaluso, J Maritano, R Chaube, F Chua, M Kokosi, V Kouranos, AU Wells, TM Maher, PM George, ER Renzoni, PL Molyneaux. Royal Brompton Hospital, London, UK

\subsection{6/thoraxinl-2017-210983.62}

Introduction There is growing evidence of the role of infection in the pathogenesis of Idiopathic pulmonary fibrosis (IPF). Azithromycin, a macrolide antibiotic, has antibacterial and antiinflammatory activity and has shown to be beneficial in animal models of lung fibrosis. This study aimed to assess the effects of prophylactic Azithromycin on hospital admissions, rescue antibiotic use and lung function in IPF.

Method A retrospective analysis identified all IPF patients receiving a prophylactic prescription of $250 \mathrm{mg}$ Azithromycin three times a week (Monday, Wednesday and Friday) between 2012 and 2017. An IPF diagnosis was made, according to international guidelines, ${ }^{1}$ following multi-disciplinary team discussion. The use of immunosuppressive therapy, immunodeficiency or the use of other prophylactic antibiotics resulted in study exclusion.

Results One hundred and fifteen patients with IPF receiving prophylactic Azithromycin were identified. Thirteen already established on therapy and 5 who received other prophylactic antibiotics were excluded. The remaining 97 IPF subjects had a mean age of $66.05 \pm 11.25$ years, were predominantly male (65\%) with moderately severe disease (DLco 34\% $\pm 9.5 \%$ predicted; FVC $70 \% \pm 18 \%$ predicted). The majority (92\%) of IPF patients tolerated Azithromycin, only 8 (8.25\%) discontinued therapy due to side effects (tinnitus $(n=1)$ and gastrointestinal intolerance $(n=7))$. One discontinued following lung transplant and 4 had therapy discontinued at the discretion of the prescribing clinician who felt there had been no subjective improvement. In the Pre-treatment twelve month period a total of 29 hospital admissions $(0.30 \pm 06$ per patient years) and 146 courses of antibiotics $(1.50 \pm 1.70$ per patient years) were recorded. In the same cohort a year after commencing prophylactic Azithromycin, there were 7 hospital admissions $(0.08 \pm 0.3$ per patient years) and 31 therapeutic antibiotic courses prescribed $(0.36 \pm 0.8$ per patient years) $(p=0.0086$, $\mathrm{p}<0.0001$ respectively) (figure 1). Lung function rate of change over the 12 months preceding and following initiation of antibiotics was examined and there was no significant change in rate of decline in either FVC or DLco.

Conclusions The present study has shown the beneficial effect of prophylactic Azithromycin in IPF patients, decreasing both hospital admissions and antibiotic usage, however, further

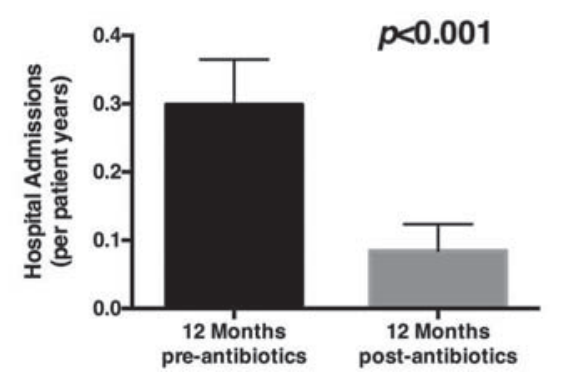

Abstarct S56 Figure 1 Comparison of additional courses of Antibiotics and hospital admissions before (black) and after (grey) the use of prophylactic Azithromycin. Data represented per patient years. 
randomised placebo controlled studies are needed to support and confirm our findings.

\section{REFERENCE}

1. Raghu G, Collard HR, Egan JJ, et al. An official ATS/ERS/JRS/ALAT statement: idiopathic pulmonary fibrosis: evidence-based guidelines for diagnosis and management. Am J Respir Crit Care Med 2011;183:788-824.

\section{S57 PREDICTORS OF UPTAKE OF AMBULATORY OXYGEN ON COMPLETION OF THE AMBOX TRIAL, A STUDY TO ASSESS EFFECTS OF AMBULATORY OXYGEN ON QUALITY OF LIFE IN PATIENTS WITH FIBROTIC INTERSTITIAL LUNG DISEASE}

${ }^{1} L$ Mori, ${ }^{2} S$ Canu, ${ }^{3} \mathrm{D}$ Visca ${ }^{4} \mathrm{~V}$ Tsipouri, ${ }^{4} \mathrm{M}$ Bonini, ${ }^{4} \mathrm{M}$ Pavitt, ${ }^{1} \mathrm{~S}$ Fleming, ${ }^{1} \mathrm{~A}$ Fiouzi, ${ }^{5} \mathrm{M}$ Farquhar, ${ }^{1} \mathrm{E}$ Leung, ${ }^{1} \mathrm{C}$ Hogben, ${ }^{6} \mathrm{~A}$ De Lauretis, ${ }^{1} \mathrm{M}$ Kokosi, ${ }^{1} \mathrm{PM}$ George, ${ }^{1} \mathrm{PL}$ Molyneaux, ${ }^{7} \mathrm{~J}$ Brown, ${ }^{8} \mathrm{~N}$ Rippon, ${ }^{9} \mathrm{~A}$ Chetta, ${ }^{1} \mathrm{AM}$ Russell, ${ }^{1} \mathrm{P}$ Saunders, ${ }^{1} \mathrm{~V}$ Kouranos, ${ }^{1} \mathrm{G}$ Margaritopoulos, ${ }^{1} \mathrm{TM}$ Maher, ${ }^{8} \mathrm{~A}$ Stockford, ${ }^{4} \mathrm{~N}$ Hopkinson, ${ }^{10} \mathrm{SS}$ Birring, ${ }^{1} \mathrm{AU}$ Wells, ${ }^{4} \mathrm{~W}$ Banya, ${ }^{8} \mathrm{H}$ Adamali, ${ }^{7} \mathrm{~L}$ Spencer, ${ }^{11} \mathrm{P}$ Sestini. ${ }^{1}$ Royal Brompton Hospital Interstitial Lung Disease Unit, London, UK; ${ }^{2}$ Sassari University, Sassari, Italy; ${ }^{3}$ Maugeri Scientific Clinical Institute, Pavia, Italy; ${ }^{4}$ Imperial College, London, UK: ${ }^{5}$ University of East Anglia, Norwich, UK; ${ }^{6}$ Garbagnate Hospital, Garbaganate Milanese, Italy; ${ }^{7}$ Aintree University, Liverpool, UK; ${ }^{8}$ North Bristol Hospital, Bristol, UK; ${ }^{9}$ Parma University, Parma, Italy; ${ }^{10}$ King's College Hospital, London, UK; ${ }^{11}$ Siena University, Siena, Italy

\subsection{6/thoraxjnl-2017-210983.63}

Background There are no ILD specific guidelines on the use of ambulatory oxygen. The AmbOx trial is a multicenter, randomised, cross-over controlled trial (NCT02286063), to assess quality of life during two weeks on ambulatory oxygen compared to two weeks off oxygen, in patients with fibrotic ILD.

Methods Individuals with fibrotic ILD whose oxygen saturation was normal at rest, but dropped to $\leq 88 \%$ on a $6 \mathrm{MWT}$, with stable symptoms during a two week run-in period, were recruited and randomised. Primary outcome: health status assessed by King's Brief ILD questionnaire (KBILD). A simple question on whether breathlessness had changed (better, same, worse) over the previous two weeks was a key secondary outcome. Patients' experiences with portable oxygen were explored through interviews in a subgroup. At the end of the four week trial period, patients were asked if they wished to continue with the ambulatory oxygen.

Results Out of 84 randomised patients, 76 completed the trial. Mean age $64.5 \pm 1.1$ years, 58 males, 53 ever smokers, FVC $73.3 \% \pm 19.1 \%$, DLCO $38.7 \% \pm 12.8 \%$. 43 patients had possible/definite IPF. Ambulatory oxygen, compared to no oxygen, was associated with improvements in total KBILD score $(p<0.0001)$. At the end of the two weeks on oxygen, the majority of patients reported improved breathlessness (better:52/76 - same:23/76 - worse:1/76), compared to the two weeks on no oxygen (better 1/76 - same:57/76 - worse:18/ 76). On trial completion, $51 / 76(67 \%)$ of patients chose to continue on ambulatory oxygen. On multivariate analysis, factors independently predictive of the patient's decision to continue, included younger age (64.8 vs 72.8 years, $\mathrm{p}=0.002)$, more severe disease (CPI 55.5 vs $49.1, \mathrm{p}=0.003$ ) and patient's global assessment of improvement in breathlessness (OR 3.2, $\mathrm{p}=0.018$ ). Despite symptomatic improvements in the majority, ambulatory oxygen was also associated with a number of patient-reported challenges, explored in the patient interviews.

\section{Paediatric asthma: big and real world data}

\section{S58 FEV1 AND FENO AS PREDICTORS OF ASTHMA OUTCOMES IN CHILDREN? AN INDIVIDUAL PATIENT DATA ANALYSIS USING DATA FROM SIX FENO TRIALS}

${ }^{1} S$ Fielding, ${ }^{2} \mathrm{M}$ Pijnenburg, ${ }^{2} \mathrm{~J}$ de Jongste, ${ }^{3} \mathrm{~K}$ Pike, ${ }^{3} \mathrm{G}$ Roberts, ${ }^{4} \mathrm{H}$ Petsky, ${ }^{4} \mathrm{~A}$ Chang, ${ }^{5} \mathrm{M}$ Fritsch, ${ }^{5} \mathrm{~T}$ Frischer, ${ }^{6} \mathrm{~S}$ Szefler, ${ }^{7} \mathrm{P}$ Gergen, ${ }^{8} \mathrm{~F}$ Vermeulen, ${ }^{8} \mathrm{R}$ Vael, ${ }^{1} \mathrm{~S}$ Turner. ${ }^{1}$ University of Aberdeen, City, UK; ${ }^{2}$ Erasmus University, Rotterdam, The Netherlands; ${ }^{3}$ University of Southampton, Southampton, UK; ${ }^{4}$ Royal Children's Hospital, Brisbane, Australia; ${ }^{5}$ Wilhelminenspital, Vienna, Austria; ${ }^{6}$ Children's Hospital Colorado, Colorado, US: ${ }^{7}$ National Institute for Allergy and Infectious Diseases, Bethseda, US; ${ }^{8}$ St Pierre University Hospital, Brussels, Belgium

\subsection{6/thoraxjnl-2017-210983.64}

Introduction Some guidelines advocate using $\mathrm{FEV}_{1}$ and/or fractional exhaled nitric oxide (FeNO) in the management of childhood asthma, but evidence supporting these recommendations is generally unsupportive. Our hypothesis was that reduced $\mathrm{FEV}_{1}$ and/or elevated FeNO measurements were associated with increased risk of future asthma attacks and loss of asthma control.

Methods Data were obtained from six trials where FeNO was used to guide asthma treatment. Baseline $\% \mathrm{FEV}_{1}$ and $\mathrm{FeNO}$ were linked to exacerbation and loss of control between baseline and three months. Change in $\% \mathrm{FEV}_{1}$ and $\%$ change in FeNO between baseline and 3 months were also linked to exacerbation and loss of control between three and six months after baseline. A one-stage individual patient data meta-analysis was conducted using a random effect for study. Baseline confounders included in the model were age, sex, LABA, LTRA, ICS dose, trial arm, control and FeNO or FEV as appropriate.

Results Data were available in 1049 children (58\% male, mean age 12.7 years) from six trials. Each unit reduction in baseline $\% \mathrm{FEV}_{1}$ was associated with increased risk for future exacerbation (OR $1.02[1.00,1.03] \mathrm{n}=935, \mathrm{p}=0.034$ ) and with increased risk for loss of control (1.01 [1.00, 1.02], $\mathrm{n}=940, \mathrm{p}=0.026$ ) after three months. Similar associations were present between change in $\% \mathrm{FEV}_{1}$ and outcomes after six months. Baseline FeNO was not related to asthma outcomes but each 10\% increase in FeNO between baseline and three months was associated with increased risk for loss of asthma control by six months (OR 1.02 [1.01, 1.03], $\mathrm{n}=725$, $\mathrm{p}=0.009$ ) but not with asthma exacerbation. Falling\% $\mathrm{FEV}_{1}$ and rising $\%$ FeNO between baseline and three months were independently associated with loss of control at six months.

Conclusions Baseline \% $\mathrm{FEV}_{1}$ is rather weakly associated with future asthma outcomes, and change in $\% \mathrm{FEV}_{1}$ between visits does not strengthen this association. In contrast, baseline FeNO is not related to future outcomes but $\%$ change in FeNO has some precision for future asthma control. The utility of $\% \mathrm{FEV}_{1}$ and $\mathrm{FeNO}$ in childhood asthma management needs to be rigorously evaluated in a clinical trial. 